Doug Geisler, Eva K. Grebel, and Dante Minniti, eds.

\title{
Memorable Quotes
}

What you call old open clusters are young clusters!

When you reach the plate limit you always have a Horizontal Branch.

Powerpoint was invented so you could blink CMDs.

There are NO globular clusters at the peak of the luminosity function!

We don't know how many globular clusters there are in the Milky Way.

Since globular clusters don't bounce, NGC 3201 (with a retrograde orbit at 250 $\mathrm{km} / \mathrm{s}$ ) had to have come in that way (and been captured).

If you are missing $10-20 \%$ (of the Galactic GCs), then you are only doing $80-90 \%$ science.

It's worth getting up at 8:30 on Wednesday morning (for my talk).

The Milky Way is doing the best it can to form massive clusters with the material that it has.

You just have to take my word for it.

The evidence for them being in circular orbits is circular.

You don't see the age gap in those diagrams because those ages are wrong.

The question is whether or not we are going to believe this.

The PSF (of the NICMOS data on 30 Doradus) looks like a Tarantula.

I'll tell you some numbers but you mustn't write them down.

I have 2 posters so please don't scream at me if I go over the 2 minute limit.

Is the evidence for dark matter in dwarf galaxies evaporating with the stars?

This is a mild-mannered but slightly eccentric galaxy. But beneath its bland exterior this galaxy is a raging psycopath.

I'm an optimistic person and I don't like bad news.

If we saw a lot of big $\mathrm{H}$ II regions in an Sa galaxy we would call it an Sc. 
Nomenclature can be defined as infant mortality rate.

Our sample is even larger and more homogeneous.

To generalize is to be an idiot.

Any GC meeting must specifically discuss the specific frequency of M87.

(After glitch with overhead projector): Whew! I was afraid I would have to move to powerpoint.

These are recent results - from last week or even this week.

When you are doing something like that (a big survey) you get a few surprises, and that's what we were after.

$\mathrm{K}$ band photometry makes you sleep better.

My collaborators, who are the people who make me work.

The low metallicity models fit best, so if I go to zero metallicity it should be a perfect fit.

Those of you in back will understand the need for angular resolution.

If we didn't know about it, we would say that this type of system could not exist.

As many of us discovered after last night (dancing), everything gets slower with age.

Adaptive optics requires small numbers of not-yet buildable lasers.

A $3.5 \mathrm{~m}$ telescope is tiny - its below the funding horizon.

I don't think we need to wait for ALMA. Some of us may not live that long.

Higher $\mathrm{S} / \mathrm{N}$ is never a mistake.

Globular clusters are special but regular. 